\title{
Editorial
}

\section{Acute Respiratory Failure: Pathophysiological Basis From A Multidisciplinary Clinical Approach}

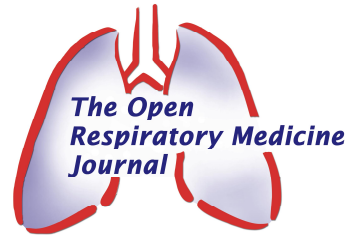

\author{
Rodrigo L. Castillo, ${ }^{*}$
}

Programa de Fisiopatología, Instituto de Ciencias Biomédicas, Facultad de Medicina, Universidad de Chile, Chile

\section{INTRODUCTION TO SPECIAL ISSUE}

\section{Acute Respiratory Failure: Pathophysiological Basis from a Multidisciplinary Clinical Approach}

Acute Respiratory Failure (ARF) is a syndrome characterized by the inability of the respiratory system to maintain adequate arterial $\mathrm{O}_{2}$ and $\mathrm{CO}_{2}$ levels according to the demands of cellular metabolism. ARF may be caused by the failure of the exchanger, lung, organ or pump, or failure of the respiratory muscles. ARF can be classified by the dysfunctional element or the evolution time when the condition occurs.

Acute lung injury/acute respiratory distress syndrome (ALI/ARDS) is the most important cause of adults hipoxemic respiratory failure, which results from multiple clinical factors such as sepsis or trauma [1]. Although the main cause was clinically resolved, the mechanism that determines the progression may persist and lead to complications.

However, It is demonstrated that the clinical causes of respiratory failure increase the intensive care units (ICU) stay, the sanitary cost and the global mortality [2]. For this reason, multidisciplinary and pathophysiological focus on respiratory failure is necessary for the management of these patients.

The application of non-invasive mechanical ventilation (NIMV) has acquired major relevance in the last few years in the management of ARF, in patients with hypoxemic and hypercapnic failure. The main advantage of NIMV compared to invasive mechanical ventilation is that it can be used earlier outside ICU. The evidence strongly supports its use in patients with chronic obstructive pulmonary disease (COPD) exacerbation, patients with acute cardiogenic pulmonary edema and immunosuppressed patients.

This issue is focused on the mechanism associated with ARF, some clinical syndromes, and the pathophysiological bases for the use of ventilatory strategies, with a multidisciplinary approach.

\footnotetext{
*Address correspondence to this author at the Programa de Fisiopatología, Instituto de Ciencias Biomédicas, Facultad de Medicina, Universidad de Chile. Independencia 1027, 8380453, Santiago, Chile;

Tel/Fax: 56-2-9786943, E-mail: rcastillo@med.uchile.cl

${ }^{\S}$ Guest Editor
}

\section{Pathophysiological Perspectives in Acute Respiratory Failure}

The pathophysiological basis of ALI/ARDS involved an activation and modulation of various inflammatory and inmune events. three themes are updated: ALI/ARS pathophysiology, the current concepts of transfusion-related acute lung injury (TRALI) and ventilatory induced lung injury (VILI). In the first review, the authors detailed the role of host in the evolution of lung inflammation to ALI/ARDS [3]. Indeed, relevant information is described respect to each animal model, reproducing key components of the injury and resolution phases of human ALI/ARDS, and provide a methodology to explore mechanisms and potential new therapies.

In the manuscript about current concepts of TRALI, Alvarez et al., provided a general view of mechanisms that lead to the development of this syndrome: immune-mediated and no immune- mediated TRALI. Indeed, the experimental TRALI models are focused in the importance of neutrophils in mediating the early immune response, and lung vascular injury [4].

In the review of VILI in ARDS, Carrasco et al., described in detail an approach to VILI focused on the effects of volutrauma that lead to lung injury and the 'mechanotransduction' mechanism. A more complete understanding about the molecular effects that physical forces could have, is essential for a better assessment of existing strategies as well as the development of new therapeutic strategies to reduce the damage resulting from VILI.

\section{Use of Noninvasive/Invasive Mechanical Ventilation with Pathophysiological Approaches}

In the review of Romero-Dapueto et al., is presented the evidence for the use of NIMV in patients with COPD exacerbation, patients with acute cardiogenic pulmonary edema, and Immunosuppressed patients [5]. Indeed, the pathophysiological evidence that supports the use of NIMV in other pathologies such as pneumonia, ARDS, and during procedures as bronchoscopy. In the last case, its use is still controversial because the results of these studies are inconclusive against the decrease in the rate of intubation or mortality. 
In the review of Cerpa et al., is described that the humidification of the airway is required in all patients with artificial airway and/or connected to invasive mechanical ventilation. Humidification devices can be Hygroscopy Heat or Heat and Moisture Exchangers, being the clinical characteristics the ones that determine which device should be chosen [6]. Indeed, It is important to select the right system to avoid the complications of deficient humidification, such as dryness of the respiratory mucosa, damage to the epithelium of the respiratory tract and airway obstruction by secretions.

Finally in the manuscript about the role of physiotherapist in management of ARF (Hidalgo et al.), and the connection and disconnection criteria are included into guidelines as professional resource in the ICU organization, with the same skills and obligations as those described in the literature for respiratory therapists.

My thanks to the authors who shared with the readers and me their views, expertise, and research (ICU; Clinica Alemana de Santiago, Laboratorio de Investigación Biomédica Hospital del Salvador, Clínica Dávila de Santiago, Hospital Clínico Metropolitano La Florida), and the reviewers whose contribution is very greatly appreciated.
Reviewers included: Farías JG, Sotomayor R, Zepeda R and Noriega $\mathrm{V}$.

I hope that you will find this special issue helpful and interesting.

\section{REFERENCES}

[1] ARDS Definition Task Force, Ranieri VM, Rubenfeld GD, et al. Acute respiratory distress syndrome: the Berlin Definition. JAMA 2012; 307: 2526-33.

[2] Bernard GR, Artigas A, Brigham KL, et al. The AmericanEuropean Consensus Conference on ARDS. Definitions, mechanisms, relevant outcomes, and clinical trial coordination. Am J Respir Crit Care Med 1994; 149: 818-24.

[3] Ware LB, Matthay MA. The acute respiratory distress syndrome. N Engl J Med 2000; 342: 1334-49.

[4] Jaworski K, Maślanka K, Kosior DA. Transfusion-related acute lung injury: a dangerous and underdiagnosed noncardiogenic pulmonary edema. Cardiol J 2013; 20: 337-44.

[5] Vital F, Ladeira M, Atallah A. Non-invasive positive pressure ventilation (CPAP or bilevel NPPV) for cardiogenic pulmonary edema. Respiratoria Cochrane Database Syst Rev 2013; 31; 5: CD005351.

[6] Branson RD, Campbell RS, Chatburn RL, Covington J. AARC clinical practice guideline. Humidification during mechanical ventilation. American Association for Respiratory Care. Respir Care 1992; 37: 887-90.

(C) Rodrigo L. Castillo; Licensee Bentham Open.

This is an open access article licensed under the terms of the Creative Commons Attribution Non-Commercial License (http://creativecommons.org/licenses/by-nc/3.0/) which permits unrestricted, non-commercial use, distribution and reproduction in any medium, provided the work is properly cited. 